\title{
Avaliação audiológica e eletrofisiológica da audição na síndrome de Landau-Kleffner
}

\author{
Audiologic and electrophysiologic evaluation in Landau-Kleffner \\ syndrome
}

\author{
Carla Gentile Matas ${ }^{1}$, Isabela Crivellaro Gonçalves ${ }^{2}$, Fernanda Cristina Leite Magliaro ${ }^{3}$, Renata Aparecida Leite ${ }^{4}$, \\ Laura Maria de Figueiredo Ferreira Guilhoto ${ }^{5}$
}

\begin{abstract}
RESUMO
Objetivo: Descrever os resultados obtidos nas avaliações audiológica e eletrofisiológica da audição, verificando a ocorrência de alterações auditivas periféricas e/ou centrais, de indivíduos com síndrome de Landau-Kleffner. Métodos: Foram submetidos à avaliação audiológica (inspeção do meato acústico externo, medidas de imitância acústica, audiometrias tonal e vocal) e eletrofisiológica da audição (potenciais evocados auditivos de curta, média e longa latência), quatro indivíduos com diagnóstico de síndrome de Landau-Kleffner, na faixa etária de nove a 19 anos, encaminhados ao Laboratório de Investigação Fonoaudiológica em Potenciais Evocados Auditivos do Curso de Fonoaudiologia da Universidade de São Paulo. Resultados: Os resultados mostraram que $100 \%$ dos indivíduos apresentaram alteração em pelo menos uma das avaliações realizadas, sendo que houve uma maior ocorrência de alterações no potencial evocado auditivo de média latência (100\% dos indivíduos apresentaram alterações). Conclusões: Observou-se uma grande ocorrência de alterações nos resultados das avaliações audiológicas e eletrofisiológicas da audição em indivíduos com síndrome de Landau-Kleffner. Enfatiza-se a importância da investigação da função auditiva destes indivíduos a fim de verificar possíveis relações entre os déficits da comunicação e alterações auditivas que possam estar presentes nessa população.
\end{abstract}

Descritores: Testes auditivos; Potenciais evocados auditivos; Síndrome de Landau-Kleffner; Transtornos da linguagem; Agnosia

(1) Professora Doutora do Curso de Fonoaudiologia do Departamento de Fisioterapia, Fonoaudiologia e Terapia Ocupacional da Faculdade de Medicina da Universidade de São Paulo - USP - São Paulo (SP) - Brasil.

(2) Pós-graduanda do Curso de Especialização em Audiologia Clínica da Faculdade de Medicina do ABC - FMABC - Santo André (SP), Brasil; Bolsista de Capacitação Técnica - TT3 pela Fundação de Amparo à Pesquisa do Estado de São Paulo - FAPESP.

(3) Pós-graduanda em Ciências da Reabilitação - Área de Concentração: Comunicação Humana do Departamento de Fisioterapia, Fonoaudiologia e Terapia Ocupacional da Faculdade de Medicina da Universidade de São Paulo - USP - São Paulo (SP) - Brasil.

(4) Pós-graduanda em Ciências da Reabilitação - Área de Concentração: Comunicação Humana do Departamento de Fisioterapia, Fonoaudiologia e Terapia Ocupacional da Faculdade de Medicina da Universidade de São Paulo - USP - São Paulo (SP) - Brasil.

(5) Doutora em Medicina; Neurologista Infantil da Divisão de Clínica Pediátrica do Hospital Universitário da Universidade de São Paulo - USP São Paulo (SP), Brasil.

Trabalho realizado no Laboratório de Investigação Fonoaudiológica em Potenciais Evocados Auditivos do Curso de Fonoaudiologia do Departamento de Fisioterapia, Fonoaudiologia e Terapia Ocupacional da Faculdade de Medicina da Universidade de São Paulo - USP - São Paulo (SP), Brasil

Auxílio à pesquisa fornecido pela Fundação de Amparo a Pesquisa do Estado de São Paulo - FAPESP, processo $n^{\circ}$ 04/06159-0.

Endereço para correspondência: Carla Gentile Matas. Av. Divino Salvador, 107/32, Planalto Paulista, São Paulo - SP, CEP 04078-010. E-mail: cgmatas@usp.br

Recebido em: 14/6/2006; Aceito em: 3/5/2007

\section{INTRODUÇÃO}

Os Transtornos Específicos do Desenvolvimento de Fala e Linguagem são quadros psiquiátricos infantis que se referem a alterações de aquisição de linguagem desde os primeiros estágios do desenvolvimento. Estas não são diretamente atribuíveis a anomalias neurológicas, anatômicas do aparelho fonador, comprometimentos sensoriais, retardo mental ou a fatores ambientais ${ }^{(1)}$.

Dentre estes transtornos tem-se a Afasia Adquirida com Epilepsia, também conhecida como Síndrome de LandauKleffner (SLK). Esta síndrome é um distúrbio neurológico sem cura, caracterizado por uma forma rara e maligna de epilepsia na infância, associado a um déficit de linguagem relacionado principalmente à compreensão da informação auditiva (agnosia auditiva) $)^{(1)}$.

Indivíduos portadores de SLK apresentam atividades elétricas anormais nos lobos temporais, área responsável pelo processamento da linguagem. A atividade epileptiforme geralmente ocorre durante o sono, com variação na intensidade e freqüência das crises convulsivas ${ }^{(2)}$. Podem apresentar distúrbios perceptuais, de atenção e de memória. Sendo assim, levanta-se a hipótese de que tais distúrbios ocorram de- 
vido a alterações nas atividades neurais, que podem acometer também a via auditiva central.

O quadro clínico da SLK é flutuante, com diferentes características durante as suas diversas fases. Seu início pode ocorrer dos 18 meses até o final da infância ${ }^{(3)}$, tendo como pico de incidência a faixa etária de dois a cinco anos, acometendo crianças de ambos os gêneros.

Doenças como inflamações desmielinizantes e neurocisticercose têm sido referidas como possíveis etiologias da SLK, embora tais achados não tenham sido confirmados, na maioria dos $\operatorname{casos}^{(4)}$. Melhoras são verificadas durante o desenvolvimento e após a administração de corticóides e anticonvulsivantes. O tratamento indicado para este distúrbio, além de medicamentoso (anticonvulsivantes e corticóides), é terapêutico (terapia fonoaudiológica), auxiliado por escola especial $^{(5)}$.

Para avaliar a via auditiva periférica, é comum a utilização de testes como a audiometria tonal, a audiometria vocal e as medidas de imitância acústica, sendo também possível a avaliação por meio das emissões otoacústicas.

A avaliação da via auditiva central pode ser realizada por meio de testes eletrofisiológicos, também denominados potenciais evocados auditivos, que são respostas bioelétricas frente à estimulação acústica. Estes potenciais evocados auditivos podem ser classificados em precoces, médios e tardios ${ }^{(6)}$.

As duas principais razões para a utilização destes testes são: determinar o limiar de detecção do sinal acústico e inferir sobre a integridade funcional e estrutural dos componentes neurais das vias auditivas ${ }^{(7)}$. Vale ressaltar que uma característica importante deste tipo de avaliação, principalmente com relação a indivíduos com distúrbios de linguagem, é que as respostas eletrofisiológicas da audição não dependem da habilidade lingüística do indivíduo e, com exceção dos potenciais tardios, não demandam um processamento cognitivo do estímulo acústico ${ }^{(8)}$.

O Potencial Evocado Auditivo de Tronco Encefálico (PEATE) é o teste eletrofisiológico mais utilizado na prática audiológica e pode ser observado nos primeiros 8 milissegundos (ms) após a apresentação do estímulo acústico, originando-se no nervo acústico e nas vias auditivas do tronco encefálico ${ }^{(9)}$.

O Potencial Evocado Auditivo de Média Latência (PEAML) consiste em uma série de ondas positivas e negativas que seguem o PEATE, presentes no período entre 10 a 80 ms após a estimulação acústica, tendo sua origem em áreas primárias do córtex auditivo ${ }^{(10)}$.

O P300, potencial de longa latência mais utilizado clinicamente, é um potencial endógeno que possui geradores nas áreas primárias e secundárias do córtex auditivo e ocorre aproximadamente em 300 ms após a apresentação do estímulo acústico. É uma resposta objetiva relacionada a aspectos fundamentais da função mental (percepção e cognição), eliciado de forma consciente, em uma tarefa de discriminação entre estímulos acústicos diferentes (estímulos raros e freqüentes), denominada paradigma “oddball”. A geração deste potencial é determinada por algumas habilidades cognitivas envolvidas no processamento da informação, como a atenção, discriminação e memória ${ }^{(11)}$.
Com relação às alterações auditivas, pesquisadores referem que o indivíduo com SLK ouve, mas não compreende ou interpreta os sons da fala e ambientais. Os resultados obtidos na avaliação audiológica de rotina (audiometrias tonal e vocal e medidas de imitância acústica), geralmente encontram-se dentro dos padrões de normalidade, embora haja dificuldade de condicionamento lúdico na audiometria tonal devido tanto a não compreensão da ordem dada pelo examinador, como a não associação do estímulo sonoro ao ato motor. As medidas de imitância acústica encontram-se dentro da normalidade, com curvas timpanométricas tipo A e reflexos acústicos ipsilaterais e contralaterais presentes ${ }^{(12)}$.

Em um estudo magnetoencefalográfico de um indivíduo com SLK, pesquisadores verificaram que todas as descargas elétricas ocorreram próximas ao córtex auditivo esquerdo e sugeriram que descargas unilaterais no córtex auditivo, ou próximas a ele, podem comprometer a discriminação auditiva no hemisfério afetado e provocar a supressão da informação auditiva proveniente do hemisfério oposto ${ }^{(13)}$.

Em pesquisa realizada, na qual foram analisados os potenciais evocados auditivos de curta, média e longa latências em cinco crianças com SLK, verificou-se a presença de alterações no potencial evocado auditivo de longa latência. Os autores concluíram que as alterações observadas, relacionadas à redução da voltagem do potencial que envolve áreas temporais previamente afetadas por descargas epileptiformes, sugerem uma disfunção permanente no córtex auditivo $\operatorname{associativo~}^{(14)}$.

Levando-se em consideração a importância da integridade do Sistema Auditivo Periférico e Central na aquisição e desenvolvimento de fala, linguagem e aprendizado, torna-se imprescindível que anormalidades auditivas sejam identificadas e tratadas precocemente nesta população, fornecendo melhores condições para o desenvolvimento de uma comunicação mais efetiva destes indivíduos com seu meio ambiente. Desta forma, este trabalho teve como objetivo descrever os resultados obtidos nas avaliações audiológica e eletrofisiológica da audição, verificando a ocorrência de alterações auditivas periféricas e/ou centrais, em indivíduos com SLK.

\section{MÉTODOS}

O material do presente estudo constou dos resultados das avaliações audiológica e eletrofisiológica da audição, obtidos em quatro indivíduos com SLK, na faixa etária de nove a 19 anos, sendo dois do sexo masculino e dois do sexo feminino.

Esse estudo foi realizado no Laboratório de Investigação Fonoaudiológica em Potenciais Evocados Auditivos do Centro de Docência e Pesquisa em Fonoaudiologia da Faculdade de Medicina da Universidade de São Paulo e aprovado pelo Comitê de Ética e Pesquisa desta instituição sob n ${ }^{\circ} 455 / 03$. Os indivíduos com SLK foram encaminhados pelo Departamento de Neurologia do Hospital Universitário da Universidade de São Paulo.

Inicialmente, foi realizada a anamnese para obtenção de dados pessoais relacionados à história pregressa da doença 
em questão, presença de fatores pré, peri e pós-natais considerados como de risco para deficiência auditiva, ocorrência de episódios de otite, purgação e queixas sobre dificuldades auditivas.

A seguir, foi realizada a inspeção do meato acústico externo com otoscópio da marca Heine e, então, iniciou-se a avaliação audiológica (audiometrias tonal e vocal e medidas de imitância acústica), realizada em cabina acústica.

$\mathrm{Na}$ audiometria tonal liminar, foram avaliadas as freqüências de 250 a $8000 \mathrm{~Hz}$ por via aérea, e 500 a $4000 \mathrm{~Hz}$ por via óssea (nas freqüências com limiares de via aérea maiores do que $20 \mathrm{~dB}$ NA). A audiometria vocal foi realizada por meio do Limiar de Reconhecimento de Fala (LRF) e Índice Percentual de Reconhecimento de Fala (IPRF).

Para ambos os procedimentos, utilizou-se o audiômetro modelo GSI 61, da marca Grason-Stadler. As medidas de imitância acústica foram realizadas com imitanciômetro Modelo GSI 33, da marca Grason-Stadler e abrangeram a timpanometria com um tom de sonda de $226 \mathrm{~Hz}$, e a pesquisa do reflexo acústico do músculo estapédio (ipsilateral e contralateral) nas freqüências de 500, 1000, 2000 e $4000 \mathrm{~Hz}$.

Após avaliação audiológica, foi realizada avaliação eletrofisiológica da audição, empregando-se como procedimentos o Potencial Evocado Auditivo de Tronco Encefálico (PEATE), o Potencial Evocado Auditivo de Média Latência (PEAML) e o Potencial Cognitivo P300, realizados com equipamento Modelo Traveler Express da marca Biologic. Inicialmente, foi feita a limpeza da pele com pasta abrasiva e os eletrodos fixados à pele do indivíduo por meio de pasta eletrolítica e fita adesiva (Micropore ${ }^{\mathrm{TM}}$ ). Foram verificados os valores da impedância dos eletrodos, devendo situar-se abaixo de 5 kohms. O estímulo acústico foi apresentado por um par de fones TDH-39, eliciando as respostas.

No PEATE, o estímulo acústico utilizado foi o click de polaridade rarefeita, apresentado monoauralmente a $80 \mathrm{~dB}$ nHL, a uma velocidade de apresentação de 19,0 clicks por segundo, duração de $0,1 \mathrm{~ms}$, sendo empregado um total de 2000 estímulos. A janela de análise foi de $10240 \mathrm{~ms}$, filtros passa-alto de $3000 \mathrm{~Hz}$ e passa baixo de $100000 \mathrm{~Hz}$, e ganho de 150000. Os eletrodos foram posicionados na fronte (Fpz) e nas mastóides direita e esquerda (M2 e M1) de acordo com a norma International Electrode System (IES)10-20, sendo considerado como eletrodo ativo, o fixado na mastóide da orelha testada; referência, o fixado na fronte e eletrodo terra, o fixado na orelha contralateral da orelha testada ${ }^{(15)}$. Foram obtidos dois registros na mesma intensidade, a fim de verificar a reprodutibilidade do traçado e, conseqüentemente, a presença das ondas. Foram verificadas as latências absolutas das ondas I, III, V, e interpicos I-III, III-V, I-V. Ao ocorrer ausência de respostas a $80 \mathrm{~dB}$ nHL, ou seja, ausência de todas as ondas, a intensidade do estímulo foi aumentada para $90 \mathrm{~dB}$ nHL.

Para a obtenção do PEAML, os eletrodos foram posicionados nas mastóides direita e esquerda (M2 e M1), nas junções têmporo-parietais direita e esquerda (C4 e C3) e na fronte (Fpz), de acordo com a norma IES 10-20, sendo considerado como eletrodos ativos os fixados nas mastóides, referência os fixados nas junções têmporo-parietais, e ele- trodo terra o fixado na fronte ${ }^{(15)}$. O estímulo utilizado foi o click apresentado monoauralmente a $70 \mathrm{~dB}$ nHL, a uma velocidade de apresentação de 10 clicks por segundo, sendo empregado um total de 1000 estímulos. A janela de análise foi de $99840 \mathrm{~ms}$, filtros passa-alto de $150.00 \mathrm{~Hz}$ e passabaixo de $10.00 \mathrm{~Hz}$, e ganho de 100.000. Os resultados do PEAML foram analisados a partir da latência e amplitude da onda $\mathrm{Pa}$, pois esta é usualmente a onda de maior amplitude e, portanto, a mais facilmente visualizada ${ }^{(7)}$. Apesar de a literatura especializada revelar que as modalidades contralaterais são as mais indicadas para analisar a onda $\mathrm{Pa}^{(16)}$, esta foi obtida, tanto nas modalidades contralaterais (C3/M2 e C4/M1), como nas ipsilaterais (C3/M1 e C4/M2). Nos casos de perda auditiva acentuada, a intensidade do estímulo foi aumentada para que se garantisse a percepção do mesmo pelo indivíduo.

Para a obtenção do P300, os eletrodos foram posicionados nas mastóides: direita e esquerda (M2 e M1), no vértex $(\mathrm{Cz})$ e na fronte (Fpz), segundo a norma IES 10-20, sendo considerado como eletrodo ativo, o fixado na mastóide da orelha testada; referência, o fixado no vértex e eletrodo terra, o fixado na fronte ${ }^{(15)}$. O estímulo acústico utilizado foi o toneburst a $75 \mathrm{~dB}$ nHL, nas freqüências de $1000 \mathrm{~Hz}$ (estímulo freqüente) e $1500 \mathrm{~Hz}$ (estímulo raro), apresentados de forma randômica pelo computador, com janela de análise de 512 ms, filtros passa-alto de $30.00 \mathrm{~Hz}$ e passa-baixo de $1.00 \mathrm{~Hz}$, e ganho de 15000 . O estímulo raro ocorreu de 15 a $20 \%$ do total de 300 estímulos, sendo que o indivíduo foi orientado a identificá-lo, contando mentalmente ou levantando a mão toda vez que este aparecesse ${ }^{(11)}$. Foram verificadas a presença e ausência deste potencial, bem como a latência do mesmo, quando presente. Nos casos de perda auditiva acentuada, a intensidade do estímulo também foi aumentada para que se garantisse a percepção do mesmo pelo indivíduo.

Foram considerados como normais os resultados que apresentaram:

I Avaliação audiológica: curva timpanométrica tipo $\mathrm{A}^{(17)}$; reflexos acústicos ipsi e contralaterais presentes nas freqüências de 500, 1000 e $2000 \mathrm{~Hz}$, entre 80 e 95 dB NA ${ }^{(18)}$; limiares tonais menores ou iguais a $25 \mathrm{~dB}$ NA; LRF igual ou até $10 \mathrm{~dB}$ acima da média dos limiares de audibilidade das freqüências de 500, 1000 e $2000 \mathrm{~Hz}$ na audiometria tonal $^{(19)}$; IRF entre $88 \%$ e $100 \%{ }^{(20)}$ na intensidade de 30 dB acima do LRF.

II PEATE: Foram analisados os valores das latências absolutas das ondas I, III e V, e latências interpicos I-III, III-V e I-V, utilizando-se como padrão de normalidade os valores propostos pelo Evoked Potential User Manual do equipamento BIO-LOGIC para crianças acima de 24 meses, que se encontram no Quadro 1.

III PEAML: Foram analisados os valores da amplitude (Na$\mathrm{Pa}) \mathrm{da}$ onda $\mathrm{Pa}$, nas diversas modalidades estudadas, sendo necessário apresentar uma diferença menor ou igual a $50 \%$ entre as amplitudes obtidas na comparação das modalidades ipsilateral e contralateral (C3/M1, C4/M2, C3/M2, C4/M1), duas a duas, para indicar normalida$\mathrm{de}^{(10)}$. Os valores das latências das ondas $\mathrm{Na}$ e $\mathrm{Pa}$ não foram considerados na análise dos resultados pelo fato 
Quadro 1. Padrão de normalidade dos valores de latência e interpicos do PEATE, para indivíduos acima de 24 meses, proposto pelo Evoked Potential User Manual

\begin{tabular}{|lcccccc|}
\hline & Onda I & Onda III & Onda V & Interpico I-III & Interpico III-V & Interpico I-V \\
\hline Média (ms) & 1,54 & 3,69 & 5,54 & 2,14 & 1,86 & 4,00 \\
Desvio Padrão (ms) & 0,11 & 0,10 & 0,19 & 0,23 & 0,14 & 0,20 \\
\hline
\end{tabular}

da via auditiva, em sua porção sub-cortical, região geradora deste potencial, encontrar-se ainda em processo de maturação em alguns indivíduos avaliados, não havendo, na literatura, valores de normalidade estabelecidos para a faixa etária de algumas crianças que compuseram a amostra desta pesquisa (abaixo de 10 anos de idade).

IV P300: Para a análise deste potencial, considerou-se o valor da latência da onda P300, utilizando valores de normalidade propostos pela literatura ${ }^{(21)}$, para cada faixa etária, conforme apresentado no Quadro 2.

Quadro 2. Padrão de normalidade dos valores de latência da onda P300, para cada faixa etária

\begin{tabular}{|cc|}
\hline Faixa Etária & Latência da onda P300 \\
\hline 5 a 16 anos & 241 a $396 \mathrm{~ms}$ \\
17 a 30 anos & 225 a $365 \mathrm{~ms}$ \\
\hline
\end{tabular}

Os resultados que não se encontravam de acordo com os critérios previamente descritos foram considerados alterados. O indivíduo foi considerado alterado quando pelo menos uma das orelhas, ou um dos lados, apresentava alteração.

\section{RESULTADOS}

Com relação aos resultados encontrados nas avaliações realizadas, observou-se que $100 \%$ dos indivíduos apresentaram alteração em pelo menos uma das avaliações. Um indivíduo (25\%) apresentou alteração na avaliação audiológica, um $(25 \%)$ no PEATE, quatro (100\%) no PEAML e três (75\%) no P300, sendo que um indivíduo $(25 \%)$ apresentou alterações concomitantes, ou seja, tanto na avaliação audiológica comportamental, como na eletrofisiológica da audição.

No que tange a avaliação audiológica, um indivíduo apresentou perda auditiva neurossensorial isolada nas freqüências de 6 e $8 \mathrm{kHz}$ na orelha esquerda.

Nos testes eletrofisiológicos os resultados obtidos foram:

- No PEATE, um indivíduo (25\%) apresentou atraso nos valores de latência absoluta da onda III e latência interpico I-III em ambas as orelhas, traçado que sugere alteração na via auditiva em tronco encefálico baixo.

- No PEAML, um indivíduo (25\%) apresentou apenas efeito orelha, outro $(25 \%)$ apenas efeito eletrodo, e dois indivíduos $(50 \%)$ apresentaram ambos os tipos de alteração (efeito orelha e eletrodo concomitantemente).

- No P300, 75\% dos indivíduos apresentaram alterações, sendo que o tipo de alteração encontrada foi o atraso na latência da onda P300.

No Quadro 3 encontram-se os resultados das avaliações audiológicas e eletrofisiológica da audição, classificados como normais e alterados para cada indivíduo avaliado. Nos Quadros 4, 5 e 6 encontram-se descritos, respectivamente, os valores das latências das Ondas I, III, V e interpicos I-III, III-V, I-V do PEATE, latências das Ondas Na, Pa e amplitude Na-Pa do PEAML e latência do P300.

Quadro 3. Resultados normais e alterados obtidos na avaliação audiológica, PEATE, PEAML e P300 nos indivíduos com SLK

\begin{tabular}{|ccccc|}
\hline Indivíduo & $\begin{array}{c}\text { Avaliação } \\
\text { Audiológica }\end{array}$ & PEATE & PEAML & P300 \\
\hline 1 & Normal & Normal & Alterado & Alterado \\
2 & Normal & Normal & Alterado & Normal \\
3 & Alterado & Normal & Alterado & Alterado \\
4 & Normal & Alterado & Alterado & Alterado \\
\hline
\end{tabular}

Quadro 4. Valores das latências absolutas das Ondas I, III, V e interpicos I-III, III-V, I-V em milissegundos (ms) do PEATE, obtidos nos indivíduos com SLK

\begin{tabular}{|c|c|c|c|c|c|c|c|}
\hline \multirow[b]{2}{*}{ Indivíduo } & \multirow[b]{2}{*}{ Orelha } & \multicolumn{6}{|l|}{ PEATE } \\
\hline & & $\begin{array}{c}\text { Onda I } \\
(\mathrm{ms})\end{array}$ & $\begin{array}{l}\text { Onda III } \\
\text { (ms) }\end{array}$ & $\begin{array}{c}\text { Onda V } \\
\text { (ms) }\end{array}$ & $\begin{array}{l}\text { Interpico I-III } \\
\text { (ms) }\end{array}$ & $\begin{array}{c}\text { Interpico III-V } \\
\text { (ms) }\end{array}$ & $\begin{array}{c}\text { Interpico I-V } \\
(\mathrm{ms})\end{array}$ \\
\hline \multirow[t]{2}{*}{1} & OD & 1.44 & 3.24 & 5.36 & 1.80 & 2.12 & 3.92 \\
\hline & OE & 1.40 & 3.24 & 5.16 & 1.84 & 1.92 & 3.76 \\
\hline \multirow[t]{2}{*}{2} & OD & 1.36 & 3.52 & 5.44 & 2.16 & 1.92 & 4.08 \\
\hline & OE & 1.44 & 3.64 & 5.64 & 2.20 & 2.00 & 4.20 \\
\hline \multirow[t]{2}{*}{3} & OD & 1.72 & 3.92 & 5.84 & 2.20 & 1.92 & 4.12 \\
\hline & $\mathrm{OE}$ & 1.60 & 3.76 & 5.76 & 2.16 & 2.00 & 4.16 \\
\hline \multirow[t]{2}{*}{4} & OD & 1.68 & 3.92 & 5.84 & 2.24 & 1.92 & 4.16 \\
\hline & OE & 1.72 & 4.08 & 5.76 & 2.36 & 1.68 & 4.04 \\
\hline
\end{tabular}


Quadro 5. Valores das latências das Ondas $\mathrm{Na}$ e Pa em milissegundos (ms) e amplitude Na-Pa em microvolts ( $\mu \mathrm{v}$ ) do PEAML, obtidos nos indivíduos com SLK

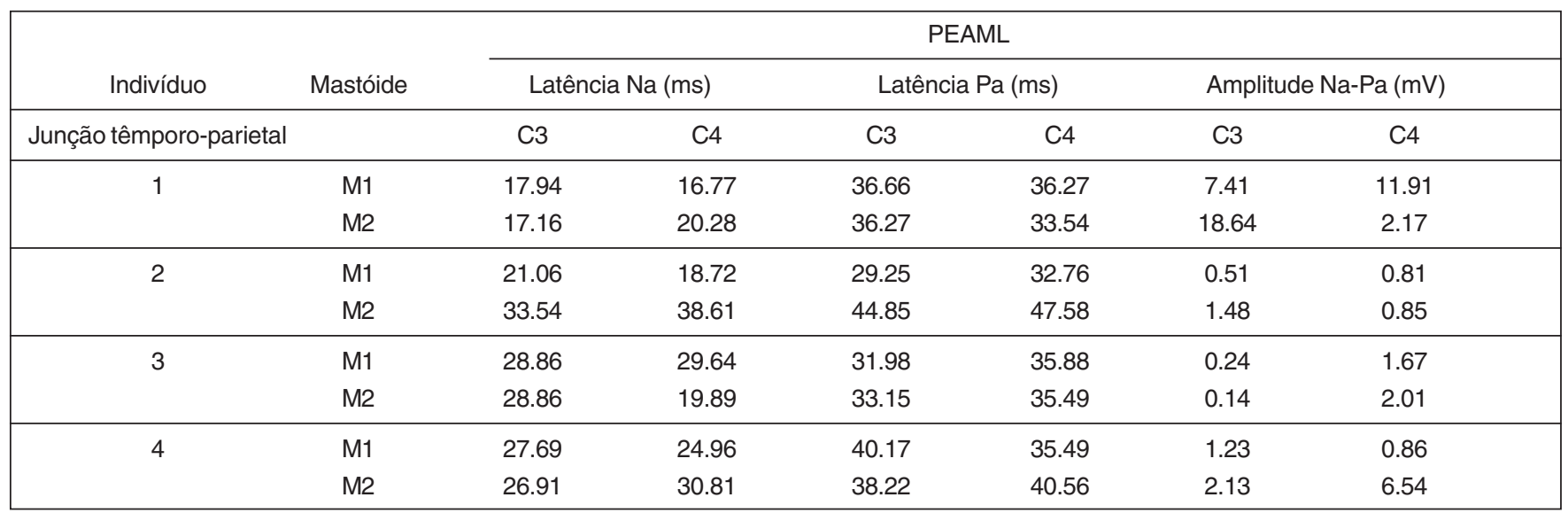

Quadro 6. Valores das latências do P300 (Potencial Cognitivo) em milissegundos ( $\mathrm{ms}$ ) obtidos nos indivíduos com SLK

\begin{tabular}{|ccc|}
\hline Indivíduo & Orelha & $\begin{array}{c}\text { Potencial Cognitivo } \\
\text { Latência P300 }(\mathrm{ms})\end{array}$ \\
\hline 1 & OD & 388 \\
& OE & 406 \\
\hline 2 & OD & 356 \\
& OE & 344 \\
\hline \multirow{2}{*}{3} & OD & 392 \\
& OE & 438 \\
\hline 4 & OD & 410 \\
& OE & 364 \\
\hline
\end{tabular}

\section{DISCUSSÃO}

Neste estudo, observamos que todos os indivíduos avaliados apresentaram alteração em algum dos procedimentos realizados. Este é um dado muito importante, pois demonstra a necessidade da realização de testes que avaliem a função auditiva de indivíduos com SLK, a fim de verificar possíveis relações entre os distúrbios da comunicação e alterações auditivas que possam estar presentes nessa população, bem como auxiliar no diagnóstico, intervenção e na definição de um melhor prognóstico para estes indivíduos.

De maneira geral, estudos demonstram que indivíduos com SLK apresentam limiares auditivos dentro da normalidade $^{(12,22-25)}$. Na presente pesquisa, verificou-se que apenas um dos quatro indivíduos $(25 \%)$ apresentou limiares auditivos rebaixados, sendo que a alteração encontrada foi do tipo neurossensorial nas freqüências altas.

Embora a literatura consultada descreva resultados normais no PEATE na SLK ${ }^{(14,24-27)}$, também encontrados em $75 \%$ dos indivíduos avaliados nesta pesquisa, observou-se que um indivíduo apresentou resultado alterado (atraso nos valores de latência absoluta da onda III e interpico I-III), sugerindo alteração na via auditiva, em tronco encefálico baixo.

No relato de caso de um paciente com SLK, também foi descrita a presença de alteração no resultado do PEATE (au- sência da onda $\mathrm{V}$ à esquerda) ${ }^{(28)}$. Considerando a alteração apontada no presente estudo e na literatura consultada ${ }^{(28)}$, deve ser enfatizada a investigação da via auditiva em tronco encefálico, visto que tais alterações podem interferir no comportamento lingüístico destes indivíduos, principalmente em relação à compreensão da informação auditiva.

No PEAML, todos os indivíduos apresentaram algum tipo de resultado alterado, sendo que a alteração de maior ocorrência foi a do tipo ambas, ou seja, dois deles (50\%) apresentaram efeito de eletrodo e de orelha, ocorrendo concomitantemente.

$\mathrm{Na}$ literatura estudada, também foram encontrados resultados discrepantes com relação ao PEAML nos indivíduos com SLK. No estudo dos potenciais evocados auditivos, em uma criança do sexo feminino de 11 anos com SLK, verificaram-se amplitudes diminuídas no PEAML ${ }^{(24)}$. Por outro lado, pesquisadores apontaram que, na realização dos potenciais evocados auditivos de curta, média e longa latências, em cinco indivíduos com SLK, as respostas de média latência encontraram-se dentro da normalidade em $100 \%$ da amostra estudada ${ }^{(14)}$.

Com relação ao P300, verificou-se uma grande ocorrência de resultados alterados (em $75 \%$ dos indivíduos avaliados), sendo que o único tipo de alteração encontrada foi o atraso na latência da onda P300.

O P300 é um potencial que está maduro somente por volta dos 14 anos de idade ${ }^{(29)}$. Na presente pesquisa, dentre os três indivíduos que apresentaram alteração neste potencial, um deles encontrava-se abaixo desta faixa etária e fora dos parâmetros de normalidade previamente estabelecidos. Estudos magnetoencefalográficos e os resultados das análises de localização das fontes elétricas no cérebro sustentam a idéia de geradores nas áreas temporais e do hipocampo para o componente $\mathrm{P} 300^{(30)}$. Considerando-se que a SLK é caracterizada por atividade epileptiforme paroxística no lobo temporal posterior $^{(2)}$, sugere-se que tais atividades possam interferir na geração deste potencial, contribuindo para a ocorrência de alterações no mesmo.

No presente estudo, não foram descritos dados em função dos valores de amplitude da onda P300, visto que tal medida é altamente variável, o que torna a efetividade deste índice incerta ${ }^{(10)}$. 
Poucos estudos são encontrados na literatura especializada que descrevam de maneira detalhada as alterações encontradas com relação aos resultados das avaliações audiológica comportamental e eletrofisiológica da audição em indivíduos com SLK, fato que dificulta a realização de uma discussão abrangente com relação aos resultados obtidos na presente pesquisa.

\section{CONCLUSÃO}

Os resultados da presente pesquisa, bem como os estudos descritos na literatura sugerem a presença de alterações audi- tivas em indivíduos com SLK, principalmente com relação aos potenciais evocados auditivos de média e longa latência.

O fonoaudiólogo tem um importante papel junto a indivíduos com SLK, não só no que tange a avaliação audiológica destes sujeitos, mas também em seu processo de reabilitação, visto que os mesmos apresentam distúrbios de linguagem significativos. Neste sentido, torna-se de suma importância a avaliação da audição, tanto em sua porção periférica, quanto central, a fim de verificar eventuais relações entre déficits de comunicação e alterações auditivas.

Deste modo, mais estudos se fazem necessários para uma melhor compreensão da função auditiva destes indivíduos.

\begin{abstract}
Purpose: To describe the audiological and electrophysiological results of individuals with Landau-Kleffner syndrome, verifying the occurrence of peripheral and/or central auditory disorders. Methods: Four individuals with Landau-Kleffner syndrome with ages ranging from nine to 19 years old, referred to the Auditory Evoked Potentials Laboratory of the Speech and Language Pathology and Audiology Course of the University of São Paulo, were submitted to audiologic (otoscopy, immitance measurements, pure tone and speech audiometry) and electrophysiological evaluation (short, middle and long-latency auditory evoked potentials). Results: All individuals showed alterations in at least one of the evaluations performed, and it was observed a higher rate of alterations in the middle latency response (100\% of the individuals showed alterations). Conclusions: A great occurrence of auditory disorders in audiological and electrophysiological evaluation of individuals with Landau-Kleffner syndrome was observed. It is emphasized the importance of the audiological investigation of these individuals, in order to verify possible relations between communication and auditory disorders in this population.
\end{abstract}

Keywords: Hearing tests; Evoked potentials, auditory; Landau-Kleffner syndrome; Language disorders; Agnosia

\title{
REFERÊNCIAS
}

1. Organização Mundial de Saúde. Classificação de transtornos mentais e de comportamento da CID - 10: descrições clínicas e diretrizes diagnósticas. Porto Alegre: Artes Médicas; 1993. p. 246-9.

2. Seri S, Cerquiglini A, Pisani F. Spike-induced interference in auditory sensory processing in Landau-Kleffner syndrome. Electroencephalogr Clin Neurophysiol. 1998;108(5):506-10.

3. Bishop DV. Age of onset and outcome in 'acquired aphasia with convulsive disorder' (Landau-Kleffner syndrome). Dev Med Child Neurol. 1985;27(6):705-12.

4. Denes G. Landau-Kleffner syndrome clinical and linguistic aspects. In: Stemmer B, Whitaker HA, editors. Handbook of neurolinguistics. San Diego: Academic Press; c1998. p. 507-14.

5. Baynes K, Kegl JA, Brentari D, Kussmaul C, Poizner H. Chronic auditory agnosia following Landau-Kleffner syndrome: a 23 year outcome study. Brain Lang. 1998;63(3):381-425.

6. Picton TW, Hillyard SA, Krausz HI, Galambos R. Human auditory evoked potentials. I. Evaluation of components. Electroencephalogr Clin Neurophysiol. 1974;36(2):179-90.

7. Kraus N, Kileny P, McGee T. Potenciais auditivos evocados de média latência (MLR). In: Katz J, editor. Tratado de audiologia clínica. 4a ed. São Paulo: Manole; 1999. p. 384- 402.

8. Schochat E. Avaliação eletrofisiológica da audição. In: Ferreira LP, BefiLopes DM, Limongi SCO, organizadores. Tratado de Fonoaudiologia. São Paulo: Roca; 2004. p. 656-68.

9. Matas CG, Leite RA, Gonçalves IC, Neves IF. Potencial evocado auditivo de tronco encefálico em indivíduos com perdas auditivas condutivas e neurossensoriais. Arq Int Otorrinolaringol. 2005;9(4):280-6.

10. Musiek FE, Lee WW. Potenciais auditivos de média e longa latência.
In: Musiek FE, Rintelmann WF, editores. Perspectivas atuais em avaliação auditiva. Barueri: Manole; 2001. p. 239-56.

11. Junqueira CAO, Colafêmina JF. Investigação da estabilidade inter e intra-examinador na identificação do P300 auditivo: análise de erros. Rev Bras Otorrinolaringol. 2002;68(4):468-78.

12. Korkman M, Granström ML, Appelqvist K, Liukkonen E. Neuropsychological characteristics of five children with the LandauKleffner syndrome: dissociation of auditory and phonological discrimination. J Int Neuropsychol Soc. 1998;4(6):566-75.

13. Paetau R, Kajola M, Korkman M, Hämäläinen M, Granström ML, Hari R. Landau-Kleffner syndrome: epileptic activity in the auditory cortex. Neuroreport. 1991;2(4):201-4.

14. Wioland N, Rudolf G, Metz-Lutz MN. Electrophysiological evidence of persisting unilateral auditory cortex dysfunction in the late outcome of Landau and Kleffner syndrome. Clin Neurophysiol. 2001;112(2):319-23.

15. Hall III JW. Handbook of auditory evoked responses. Boston: Allyn and Bacon; 1992.

16. Kimura D. Some effects of temporal-lobe damage on auditory perception. Can J Psychol. 1961;15:156-65.

17. Jerger J. Clinical experience with impedance audiometry. Arch Otolaryngol. 1970;92(4):311-24.

18. Carvallo RMM, Carvalho M, Ishida IM. Auditory profile in individuals with and without CAPD. In: American Academy of Audiology Annual Convention \& Exposition, 12th São Paulo: American Academy of Audiology Annual Convention \& Exposition; 2000. p.150.

19. Santos TMM, Russo ICP. Logoaudiometria. In: Santos TMM, Russo ICP. A prática da audiologia clínica. 3a ed. São Paulo: Cortez; 1991. p.73-88. 
20. Gates GA, Chakeres DW; American Academy of Otolaryngology-Head and Neck Surgery Foundation. Interpretation of diagnostic tests for acoustic neuroma. Washington, D.C.: American Academy of Otolaryngology-Head and Neck Surgery Foundation; 1988.

21. McPherson DL. Late potentials of the auditory system. San Diego: Singular; c1996.

22. Kale U, el-Naggar M, Hawthorne M. Verbal auditory agnosia with focal EEG abnormality: an unusual case of a child presenting to an ENT surgeon with "deafness". J Laryngol Otol. 1995;109(5):431-2.

23. Stroink H, Van Dongen HR, Meulstee J, Scheltens-de Boer M, Geesink HH. [A special case of 'deafness'; Landau-Kleffner syndrome]. Ned Tijdschr Geneeskd. 1997;141(33):1623-5. Dutch.

24. Zouari N, Choyakh F. [Early, middle-latency and late auditory evoked potentials in a case of acquired epileptic aphasia (Landau-Kleffner syndrome)]. Rev Laryngol Otol Rhinol (Bord). 1997;118(4):267-70. French.
25. Erdem T, Kirazli T, Tutuncuoglu S. [Landau-Kleffner syndrome (acquired epileptic aphasia)]. Kulak Burun Bogaz Ihtis Derg. 2003;10(1):25-8. Turkish.

26. Koeda T, Kohno Y. [Non-verbal auditory agnosia with EEG abnormalities and epilepsy; an unusual case of Landau-Kleffner syndrome]. No To Hattatsu. 1992;24(3):262-7. Japanese.

27. Denes G, Balliello S, Volterra V, Pellegrini A. Oral and written language in a case of childhood phonemic deafness. Brain Lang. 1986; 29(2):25267.

28. Matas CG, Scheuer C, Magliaro FCL, Ogata JE, Leite RA. Audiometria de tronco encefálico e síndrome de Landau-Kleffner: estudo de caso. Acta AWHO. 2002;21(3/4).

29. Buchwald JS. Comparison of plasticity in sensory and cognitive processing systems. Clin Perinatol. 1990;17(1):57-66.

30. Tarkka IM, Stokic DS, Basile LF, Papanicolaou AC. Electric source localization of the auditory P300 agrees with magnetic source localization. Electroencephalogr Clin Neurophysiol. 1995;96(6):538-45. 